\title{
AVALIAÇÃO DOS CUSTOS DE IMPLANTAÇÃO DE BIODIGESTORES E DA ENERGIA PRODUZIDA PELO BIOGÁS
}

Doi:http://dx.doi.org/10.1590/1809-4430-Eng.Agric.v35n6p 990-997/2015

\section{LANA F. CALZA ${ }^{1}$, CLEB ER B. LIMA ${ }^{2}$, CARLOS E. C. NOGUEIRA ${ }^{3}$, JAIR A. C. SIQUEIRA ${ }^{4}$, REGINALDO F. SANTOS ${ }^{5}$}

\begin{abstract}
RESUMO: Este trabalho objetivou avaliar os custos envolvidos na construção de modelos de biodigestores (indiano, chinês e canadense) no município de Cascavel - PR. Determinaram-se os custos de produção de energia a partir do biogás produzido por dejetos de caprinos, bovinos e suínos, para sistemas de semiconfinamento. Foram utilizadas propriedades rurais com 20;40;60; 80 e 100 animais de cada um dos três grupos avaliados. A partir do volume de dejetos produzidos por animal, foram dimensionados os biodigestores e calculados os custos para a construção e a implantação de cada modelo, e o custo total da produção anual de energia em função do número de animais. O biodigestor modelo canadense apresentou o menor custo de construção e de implantação, variando de $\mathrm{R}$ \$ 2.104,00 a R\$ 7.266,00. A geração de energia com dejetos de suínos apresentou o menor custo, variando de 0,015 a $0,050 \mathrm{R} \$ / \mathrm{KWh}$ em biodiges tor modelo canadense. A maior produção anual de energia foi verificada para bovinos, variando de $24.090 \mathrm{kWh} / \mathrm{ano}$ a $120.450 \mathrm{kWh} / \mathrm{ano}$, para os três modelos de biodigestor em estudo.
\end{abstract}

PALAVRAS-CHAVE: biogás, esterco, a valiação econômica.

\section{COST ASSESSMENT OF BIODIGESTER IMPLEMENTATION AND BIOGAS- PRODUCED ENERGY}

\begin{abstract}
This study aimed at assessing the costs incurred in building different biodigester models (Indian, Chinese, and Canadian) in Cascavél-PR, Brazil. We also determined costs related to biogas production from goat, bovine, and swine dungs in semi-confinement systems. For this purpose, we evaluated farms with herds of 20,40, 60, 80, and 100 animals of each group. The biodigesters' volume were based on each animal amount of excrement and, then, we calculated the costs for building and implementing each model, as well as costs associated to annual energy production on the number of animals used. The lowest cost was observed for Canadian biodigester with respect to both building and implementation, ranging from $\mathrm{R} \$ 2,104.00$ to $\mathrm{R} \$ 7,266.00$. Power generation using swine waste had the lowest costs varying 0.015 to $0.050 \mathrm{R} \$ / \mathrm{kWh}$ with Canadian model. The largest annual energy production was recorded for bovine dung, varying 24,090 to $120,450 \mathrm{kWh} /$ year for the 3 studied models.
\end{abstract}

KEYWORDS: biogas, manure, economic assessment.

\footnotetext{
${ }^{1}$ Eng $^{\mathrm{a}}$ Civil, Acadêmica Mestre, Programa de Pós-Graduação em Engenharia de Energia na Agricultura, UNIOESTE/Cascavel-PR, Fone: (45) 3220-3151, lanacalza.eng@ gmail.com

${ }^{2}$ Eng $^{\circ}$ A grícola, Acadêmico Graduado, Programa de Pós-Graduação em Engenharia de Energia na Agricultura, UNIOESTE/Cascavel-PR, maguila_333@hotmail.com

${ }^{3}$ Eng $^{\circ}$ A grícola e Eletricista, Prof. Doutor, Programa de Pós-Graduação em En genharia de Energia na A gricultura, UNIOESTE/Cascavel-PR, carlos.nogueira@unioeste.br

${ }^{4}$ Eng ${ }^{\circ}$ A grícola, Prof. Doutor, Programa de Pós-Graduação em En genharia de Energia na A gricultura, UNIOESTE/Cascavel-PR, jair.siqueira@unioeste.br

${ }^{5}$ Eng $^{\circ}$ A grônomo, Prof. Doutor, Programa de Pós-Graduação em En genharia de Energia na A gri cultura, UNIOESTE/Cascavel-PR, reginaldo.santos@unioeste.br 


\section{INTRODUÇÃO}

No Brasil, os resíduos de origem animal constituem-se importante fonte de obtenção da biomassa, e a sua utilização em sistemas biointegrados para fins energéticos mostra-se favorável sob aspectos econômicos e ambientais.

O constante aumento das atividades agrícolas tem resultado em um considerável aumento na produção de dejetos, ocasionando problemas de ordem sanitária pela grande quantidade de organismos patogênicos presentes nesses resíduos, e pela contaminação da água e do solo.

De acordo com DHANALAKSHMI \& RAMANUJAM (2012), existem diversos processos para o tratamento destes resíduos, onde a biodigestão anaeróbia se traduz em uma tecnologia eficiente, uma vez que permite a obtenção do biogás e do biofertilizante, cuja disponibilidade, além de contribuir para amortizar o custo de instalação da tecnologia, soluciona o problema de saneamento da disposição destes resíduos no meio ambiente.

O biogás trata-se de uma mistura gasosa com potencial combustível, composta principalmente por metano $\left(\mathrm{CH}_{4}\right)$ e gás carbônico $\left(\mathrm{CO}_{2}\right)$, que pode ser utilizado na geração de energia elétrica e no aquecimento. $\mathrm{O}$ biofertilizante trata-se de um fertilizante natural rico em nitrogênio (BALMANT, 2009). A geração de energia elétrica pode ser feita pela queima do biogás em turbinas, microturbinas e em motores do ciclo Otto e diesel, devidamente adaptados, sendo considerada uma fonte energética limpa e apropriada ao uso em propriedades rurais.

Existem vários tipos de biodigestores utilizados para a produção do biogás, conforme os tipos de resíduos obtidos no meio rural, podendo ser operados com cargas contínuas ou batelada (LOPES et al, 2003).

Segundo XAVIER \& LUCAS JÚNIOR (2010), os biodigestores operados em batelada são de construção mais simples em relação aos outros, porém faz-se necessária a análise preliminar do projeto para a criação de sistemas de biodigestores visando à construção de instalações mais econômicas com garantia de recuperação do investimento de forma rápida.

A literatura apresenta como principais modelos de biodigestor os modelos chinês, indiano e canadense, sendo que o modelo de biodigestor canadense é diferenciado dos demais por ser horizontal e por apresentar uma caixa de carga revestida com manta plástica.

CERVI et al. (2010) afirmam que a criação de fontes de suprimento descentralizadas é fundamental para o desenvolvimento sustentável, corroborando SILVA et al. (2008), que afirmam que uma das vantagens do uso do biogás é a redução da necessidade de importação de energia. SALOMON \& LORA (2009) afirmam ainda que, além da vantagem de utilizar a energia no local onde o gás é produzido, a energia excedente pode, em alguns casos, ser vendida à rede elétrica local.

Este trabalho teve como objetivo apresentar os parâmetros utilizados para a mensuração dos custos de implantação de diferentes tipos de biodigestor (chinês, indiano e canadense) para diferentes quantidades e grupos animais (caprinos, bovinos e suínos), na cidade de Cascavel - PR, bem como apresentar os custos da produção de energia a partir do biogás.

\section{MATERIAL E MÉTODOS}

Para a produção de dejetos, foram consideradas propriedades com 20;40;60;80 e 100 animais de três diferentes espécies (caprinos, bovinos e suínos), em sistemas de semiconfinamento.

De acordo com OLIVER et al. (2008), considera-se que a produção diária de dejetos para caprinos, bovinos e suínos seja, respectivamente, de 0,$5 ; 15$ e $4 \mathrm{~kg}$ de dejetos por animal. A diluição corresponde à relação esterco:água de 1:4, 1:1 e 1:1,3 para caprinos, bovinos e suínos, respectivamente. $\mathrm{O}$ tempo de retenção considerado foi de 45 dias para caprinos e 35 dias para bovinos e suínos. 
De acordo com FONSECA et al. (2009), para o dimensionamento adequado da capacidade do biodigestor, é possível utilizar um método prático de cálculo, desde que se tenha a carga diária de matéria orgânica colocada no digestor e o tempo de retenção:

$\mathrm{VB}=\mathrm{VC} \times \mathrm{TRH}$

em que,

$\mathrm{VB}=$ Volume do biodiges tor $\left(\mathrm{m}^{3}\right)$;

$\mathrm{VC}=$ Volume de carga diária (matéria orgânica + água ) ( $\left.\mathrm{m}^{3} / \mathrm{dia}\right)$,

$\mathrm{TRH}=$ tempo de retenção hidráulica (dias).

\section{Construção de biodigestores modelo Indiano e Chinês}

O biodigestor modelo indiano pode ser descrito como um cilindro vertical, construído com tijolos e revestido internamente por cimento impermeabilizante, tendo uma parede longitudinal que o divide em duas câmaras. Em uma dessas câmaras, é conectado o tubo de entrada da biomassa, e na outra, o tubo de saída. Este biodigestor caracteriza-se por possuir uma campânula flutuante como gasômetro feita de chapa de aço (NISHIMURA, 2009).

O biodigestor modelo chinês não possui gasômetro. Este biodigestor é cons truído totalmente enterrado no solo, é feito de alvenaria de tijolos e possui teto em forma de abóboda. O custo de construção é inferior ao modelo indiano por não necessitar de gasômetro e por utilizar materiais de menor custo (NISHIMURA, 2009).

Os materiais utilizados para a construção dos biodigestores estão apresentados em LUCAS JÚNIOR et al. (2003). O custo de material das caixas de carga e de descarga pode ser considerado como sendo $20 \%$ do custo de material do biodigestor. Para o biodigestor indiano, o cus to da cúpula de metal que forma o gasômetro foi calculado como sendo $25 \%$ da soma dos custos de material do biodigestor (incluindo as caixas de carga e de descarga), mão de obra e retroescavador (LUCAS JÚNIOR et al., 2003).

\section{Construção de biodigestor modelo Canadense}

Este modelo de biodigestor é caracterizado por possuir uma base retangular construída de alvenaria e um gasômetro feito em manta flexível de PVC, fixa sobre uma valeta de água que circunda a base. A cobertura é feita com geomembrana sintética de polietileno de alta densidade (PEAD), ancorada ao redor do perímetro de todo o biodigestor.

\section{Custos de construção dos biodigestores}

Os custos unitários dos materiais utilizados para a construção dos biodigestores modelo chinês, indiano e canadense foram consultados nas tabelas de índices da construção civil do Sistema Nacional de Pesquisa de Custos e Índices da Construção Civil, para o mês de setembro de 2012 (SINAPI, 2012).

Foi realizada a composição de custos para cada um dos modelos de biodigestor para diferentes faixas de capacidade, variando em 20;40;60; 80;100 e $120 \mathrm{~m}^{3}$. A escolha da capacidade do biodigestor para cada volume dos dejetos de caprinos, bovinos e suínos foi feita adotando-se o critério de que se deve escolher o biodigestor com capacidade imediatamente acima do calculado quando este não pertencer às faixas de capacidade, ou seja, o biodigestor pode ter capacidade superior à calculada, porém não poderá ter capacidade inferior.

Para a composição dos custos de construção dos biodigestores modelos indiano, chinês e canadense, foi considerado o custo com materiais de cons trução, escavação e mão de obra.

\section{Produção do biogás e energia produzida}

A estimativa da produção diária de biogás a partir de dejetos de diferentes tipos de animais foi calculada através dos dados fornecidos por OLIVER et al. (2008), correspondendo a 0,0305 
$\mathrm{m}^{3} /$ animal para caprinos, $0,6 \mathrm{~m}^{3} /$ animal para bovinos e $0,356 \mathrm{~m}^{3} / \mathrm{animal}$ para suínos. A partir deste volume de biogás produzido e do poder calorífico do biogás $\left(5,5 \mathrm{kWh} / \mathrm{m}^{3}\right)$, calculou-se a quantidade de energia a ser produzida anualmente.

O cálculo da energia elétrica produzida pelo biogás foi realizado conforme FONSECA et al. (2009) e considera o custo anualizado dos biodigestores (taxa de juros de $10 \%$ ao ano, vida útil de 20 anos, manutenção e operação anual de 5\% dos custos de implantação) e a produção anual de biogás. Foi calculado o custo total da energia produzida em função do tipo de biodigestor e da quantidade e do grupo de animais.

\section{RESULTADOS E DISCUSSÃO}

De acordo com os dados de produção de dejetos e do potencial de produção de biogás por animal, foram calculados as quantidades de dejetos produzidos para os três grupos de animais e o potencial de produção de biogás (PB). Os resultados estão apresentados na Tabela 1.

TABELA 1. Cálculo da capacidade do biodigestor e estimativa da produção de (PB) para caprinos, bovinos e suínos. Calculation of digester capacity and estimated energy production (GP) for each used dung (goat, cattle, and s wine).

\begin{tabular}{ccccccc}
\hline \multirow{2}{*}{\begin{tabular}{c} 
Quantidade de $\begin{array}{c}c \\
\text { Anima is }\end{array}$ \\
\cline { 2 - 7 }
\end{tabular}} & $\begin{array}{c}\text { Capacidade do } \\
\text { Biodigestor } \\
\left(\mathrm{m}^{3}\right)\end{array}$ & $\begin{array}{c}\text { Produção de } \\
\text { Biogás -PB } \\
\left(\mathrm{m}^{3} / \text { dia }\right)\end{array}$ & $\begin{array}{c}\text { Capacidade do } \\
\text { Biodigestor }\left(\mathrm{m}^{3}\right)\end{array}$ & $\begin{array}{c}\text { Produção de } \\
\text { biogás - PB } \\
\left(\mathrm{m}^{3} / \text { dia }\right)\end{array}$ & $\begin{array}{c}\text { Capacidade do } \\
\text { Biodigestor } \\
\left(\mathrm{m}^{3}\right)\end{array}$ & $\begin{array}{c}\text { Produção de } \\
\text { biogás - PB } \\
\left(\mathrm{m}^{3} / \mathrm{dia}\right)\end{array}$ \\
\hline 20 & 2,25 & 0,61 & 21 & 12 & 6,44 & 7,12 \\
40 & 4,50 & 1,22 & 42 & 24 & 12,88 & 14,24 \\
60 & 6,75 & 1,83 & 63 & 36 & 19,32 & 21,36 \\
80 & 9,00 & 2,44 & 84 & 48 & 25,76 & 28,48 \\
100 & 11,25 & 3,05 & 105 & 60 & 32,20 & 35,60 \\
\hline
\end{tabular}

Utilizando-se de regras geométricas simples, calculou-se a quantidade dos materiais utilizados na construção dos diferentes modelos de biodigestores com capacidades de 20;40;60;80;100 e $120 \mathrm{~m}^{3}$, para a obtenção dos custos de construção de cada um deles. Estes custos estão apresentados na Tabela 2.

TABELA 2. Custo de construção e de implantação de biodigestores modelos Indiano, Chinês e Canadense para diferentes capacidades. Building and implementation costs of Indian, Chinese, and Canadian biodigester models at varied volumes.

\begin{tabular}{cccc}
\hline \multirow{2}{*}{ Capacidade do Biodigestor $\left(\mathrm{m}^{3}\right)$} & \multicolumn{3}{c}{ Custo total por mode lo de biodigestor $(\mathrm{R} \$)$} \\
\cline { 2 - 4 } & Indiano & Chinês & Canadense \\
\hline 20 & $5.065,70$ & $4.052,50$ & $2.104,00$ \\
40 & $7.099,30$ & $5.679,40$ & $3.162,00$ \\
60 & $9.440,20$ & $7.552,10$ & $4.188,00$ \\
80 & $11.470,90$ & $9.176,70$ & $5.214,00$ \\
100 & $12.486,30$ & $10.801,40$ & $6.240,00$ \\
120 & $14.178,60$ & $12.426,00$ & $7.266,00$ \\
\hline
\end{tabular}

A partir dos dados apresentados na Tabela 2, pode-se afirmar que o biodigestor modelo canadense é o que apresenta menor custo para maior capacidade de animais atendidos, assim como o modelo indiano apresenta o maior custo de implantação para pequenos volumes.

Para caprinos, o modelo de biodigestor canadense é o mais indicado, uma vez que, para 100 animais desta espécie, o volume do biodigestor calculado é de 11,25 $\mathrm{m}^{3}$. Este volume enquadra-se na capacidade de $20 \mathrm{~m}^{3}$, sendo que o modelo canadense apresenta menor custo de construção (R\$ 2.104,00). 
Para bovinos, o modelo de biodigestor canadense é o mais indicado, uma vez que, para 100 animais desta espécie, o volume do biodigestor calculado é de 105,0 $\mathrm{m}^{3}$. Este volume enquadra-se na capacidade de $120 \mathrm{~m}^{3}$, sendo que o modelo canadense apresenta menor custo de construção (R\$ $7.266,00)$.

Para suínos, o modelo de biodigestor canadense é o mais indicado, uma vez que, para 100 animais desta espécie, o volume do biodigestor calculado é de $32,2 \mathrm{~m}^{3}$. Este volume enquadra-se na capacidade de $40 \mathrm{~m}^{3}$, sendo que o modelo canadense apresenta menor custo de construção (R\$ $3.162,00)$.

A análise dos dados permite identificar que existe um padrão na escolha do modelo do biodigestor a partir dos custos de construção e implantação, em que, independentemente da capacidade do biodigestor, o modelo indiano é o que apresenta maior custo de construção, e o modelo canadense é o biodigestor que apresenta menor custo de produção de energia a partir do biogás.

A Tabela 3 apresenta o custo anualizado (CA) da construção e da implantação dos biodigestores modelos indiano, chinês e canadense para as capacidades de $20 ; 40 ; 60 ; 80 ; 100$ e 120 $\mathrm{m}^{3}$.

TABELA 3. Custo anualizado (CA) para construção e implantação de biodigestores modelo Indiano, Chinês e Canadense. B uilding and implementation yearly costs (YC) for Indian, Chinese, and Canadian biodigester models.

\begin{tabular}{cccc}
\hline \multirow{2}{*}{ Capacidade do Biodigestor $\left(\mathrm{m}^{3}\right)$} & \multicolumn{3}{c}{ Custo total anualizado (R\$) } \\
\cline { 2 - 4 } & Indiano & Chinês & Canadense \\
\hline 20 & $1.796,60$ & $1.437,30$ & 746,20 \\
40 & $2.517,80$ & $2.014,00$ & $1.121,80$ \\
60 & $3.348,00$ & $2.678,40$ & $1.485,70$ \\
80 & $4.068,30$ & $3.254,60$ & $1.849,60$ \\
100 & $4.428,40$ & $3.830,80$ & $2.213,40$ \\
120 & $5.028,60$ & $4.407,00$ & $2.577,30$ \\
\hline
\end{tabular}

A Tabela 4 apresenta o custo total de produção de energia a partir do biogás (CTB) para o biodigestor modelo indiano para caprinos, bovinos e suínos.

TABELA 4. Custo total da produção de energia a partir do biogás (CTB) para biodigestor modelo Indiano. Biogas-produced energy total costs (CTB) for Indian biodigester model.

\begin{tabular}{cccccccccc}
\hline \multirow{2}{*}{$\begin{array}{c}\text { Quant. de } \\
\text { Animais }\end{array}$} & $\begin{array}{c}\mathrm{CA} \\
(\mathrm{R} \$)\end{array}$ & $\begin{array}{c}\mathrm{PB} \\
(\mathrm{kWh})\end{array}$ & $\begin{array}{c}\mathrm{CTB} \\
(\mathrm{R} \$ / \mathrm{kWh})\end{array}$ & $\begin{array}{c}\mathrm{CA} \\
(\mathrm{R} \$)\end{array}$ & $\begin{array}{c}\mathrm{PB} \\
(\mathrm{kWh})\end{array}$ & $\begin{array}{c}\mathrm{CTB} \\
(\mathrm{R} \$ / \mathrm{kWh})\end{array}$ & $\begin{array}{c}\text { CA } \\
(\mathrm{R} \$)\end{array}$ & $\begin{array}{c}\text { PB } \\
(\mathrm{kWh})\end{array}$ & $\begin{array}{c}\text { CTB } \\
(\mathrm{R} \$ / \mathrm{kWh})\end{array}$ \\
\hline 20 & $1.796,60$ & 1224,58 & 1,47 & $2.517,80$ & 24090 & 0,105 & $1.796,60$ & 14293,4 & 0,1260 \\
40 & $1.796,60$ & 2449,15 & 0,73 & $3.348,00$ & 48180 & 0,069 & $1.790,60$ & 28586,8 & 0,0630 \\
60 & $1.796,60$ & 3673,73 & 0,49 & $4.068,30$ & 72270 & 0,056 & $1.790,60$ & 42880,2 & 0,0420 \\
80 & $1.796,60$ & 4898,3 & 0,37 & $4.428,40$ & 96360 & 0,046 & $2.517,80$ & 57173,6 & 0,0440 \\
100 & $1.796,60$ & 6122,88 & 0,29 & $5.028,60$ & 120450 & 0,042 & $2.517,80$ & 71467,0 & 0,0352 \\
\hline
\end{tabular}

A Tabela 5 apresenta o custo total de produção de energia a partir do biogás para o biodigestor modelo chinês para caprinos, bovinos e suínos. 
TABELA 5. Custo total da produção de energia a partir do biogás (CTB) para biodigestor modelo Chinês. B iogas-produced energy total costs (CTB) for Chinese biodigester model.

\begin{tabular}{cccccccccc}
\hline \multirow{3}{*}{$\begin{array}{c}\text { Quant. de } \\
\text { Animais }\end{array}$} & \begin{tabular}{c}
$\mathrm{CA}$ \\
\cline { 2 - 10 }
\end{tabular} & $\begin{array}{c}\mathrm{PB} \\
(\mathrm{kWh})\end{array}$ & $\begin{array}{c}\mathrm{CTB} \\
(\mathrm{R} \$ / \mathrm{kWh})\end{array}$ & $\begin{array}{c}\mathrm{CA} \\
(\mathrm{R} \$)\end{array}$ & $\begin{array}{c}\mathrm{PB} \\
(\mathrm{kWh})\end{array}$ & $\begin{array}{c}\text { CTB } \\
(\mathrm{R} \$ / \mathrm{kWh})\end{array}$ & $\begin{array}{c}\mathrm{CA} \\
(\mathrm{R} \$)\end{array}$ & $\begin{array}{c}\text { PB } \\
(\mathrm{kWh})\end{array}$ & $\begin{array}{c}\mathrm{CTB} \\
(\mathrm{R} \$ / \mathrm{kWh})\end{array}$ \\
\hline 20 & $1.437,00$ & 1224,58 & 1,17 & $2.014,00$ & 24090 & 0,080 & $1.437,00$ & 14293,4 & 0,100 \\
40 & $1.437,00$ & 2449,15 & 0,59 & $2.678,40$ & 48180 & 0,060 & $1.437,00$ & 28586,8 & 0,050 \\
60 & $1.437,00$ & 3673,73 & 0,39 & $3.254,60$ & 72270 & 0,050 & $1.437,00$ & 42880,2 & 0,034 \\
80 & $1.437,00$ & 4898,30 & 0,29 & $3.830,80$ & 96360 & 0,040 & $2.014,00$ & 57173,6 & 0,030 \\
100 & $1.437,00$ & 6122,89 & 0,24 & $4.407,00$ & 120450 & 0,037 & $2.014,00$ & 71467,0 & 0,028 \\
\hline
\end{tabular}

A Tabela 6 apresenta o custo total de produção de energia a partir do biogás para o biodigestor modelo canadense para caprinos, bovinos e suínos.

TABELA 6. Custo total da produção de energia a partir do biogás (CTB) para biodigestor modelo Canadense. Biogas-produced energy total costs (CTB) for Canadian biodigester model.

\begin{tabular}{cccccccccc}
\hline & \multicolumn{3}{c}{ Caprinos } & \multicolumn{3}{c}{ Bovinos } & \multicolumn{3}{c}{ Suínos } \\
\cline { 2 - 10 } $\begin{array}{c}\text { Quant. de } \\
\text { Animais }\end{array}$ & $\begin{array}{c}\mathrm{CA} \\
(\mathrm{R} \$)\end{array}$ & $\begin{array}{c}\mathrm{PB} \\
(\mathrm{kWh})\end{array}$ & $\begin{array}{c}\text { CTB } \\
(\mathrm{R} \$ / \mathrm{kWh})\end{array}$ & $\begin{array}{c}\text { CA } \\
(\mathrm{R} \$)\end{array}$ & $\begin{array}{c}\mathrm{PB} \\
(\mathrm{kWh})\end{array}$ & $\begin{array}{c}\text { CTB } \\
(\mathrm{R} \$ / \mathrm{kWh})\end{array}$ & $\begin{array}{c}\text { CA } \\
(\mathrm{R} \$)\end{array}$ & $\begin{array}{c}\text { PB } \\
(\mathrm{kWh})\end{array}$ & $\begin{array}{c}\text { CTB } \\
(\mathrm{R} \$ / \mathrm{kWh})\end{array}$ \\
\hline 20 & 746,20 & 1224,58 & 0,61 & $1.121,80$ & 24090 & 0,05 & 746,20 & 14293,4 & 0,050 \\
40 & 746,20 & 2449,15 & 0,31 & $1.485,70$ & 48180 & 0,03 & 746,20 & 28586,8 & 0,026 \\
60 & 746,20 & 3673,73 & 0,20 & $1.849,60$ & 72270 & 0,025 & 746,20 & 42880,2 & 0,017 \\
80 & 746,20 & 4898,3 & 0,15 & $2.213,40$ & 96360 & 0,022 & $1.121,80$ & 57173,6 & 0,019 \\
100 & 746,20 & 6122,88 & 0,12 & $2.577,30$ & 120450 & 0,021 & $1.121,80$ & 71467,0 & 0,015 \\
\hline
\end{tabular}

As Figuras 1, 2 e 3 apresentam os custos totais da produção de energia a partir do biogás (CTB) em função do número de animais de caprinos, bovinos e suínos.

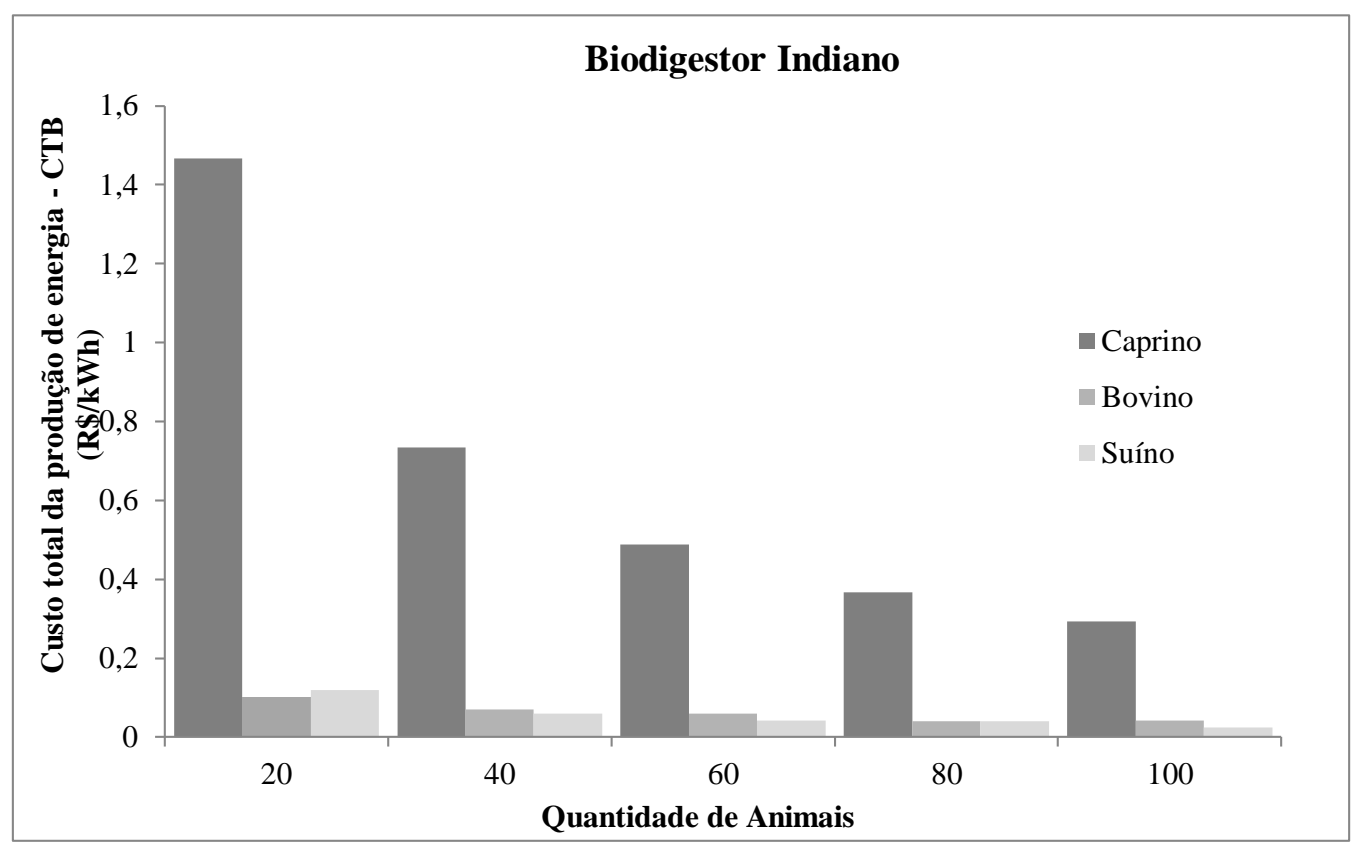

FIGURA 1. Custo total da produção de energia a partir do biogás (CTB) para biodigestor modelo Indiano. Biogas-produced energy total costs (CTB) for Indian biodigester model. 


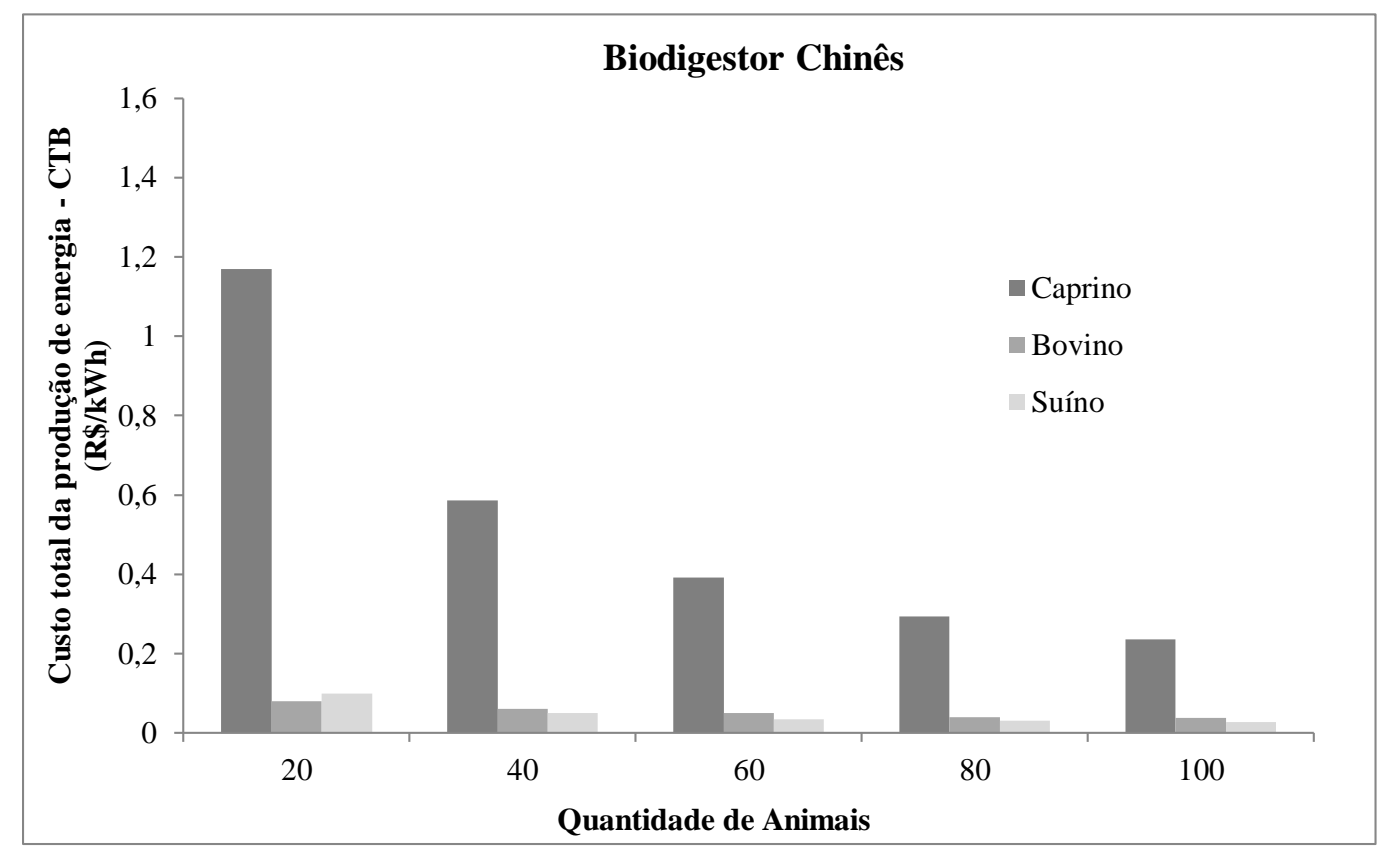

FIGURA 2. Custo total da produção de energia a partir do biogás (CTB) para biodigestor modelo Chinês. B iogas-produced energy total costs (CTB) for Chinese biodigester model.

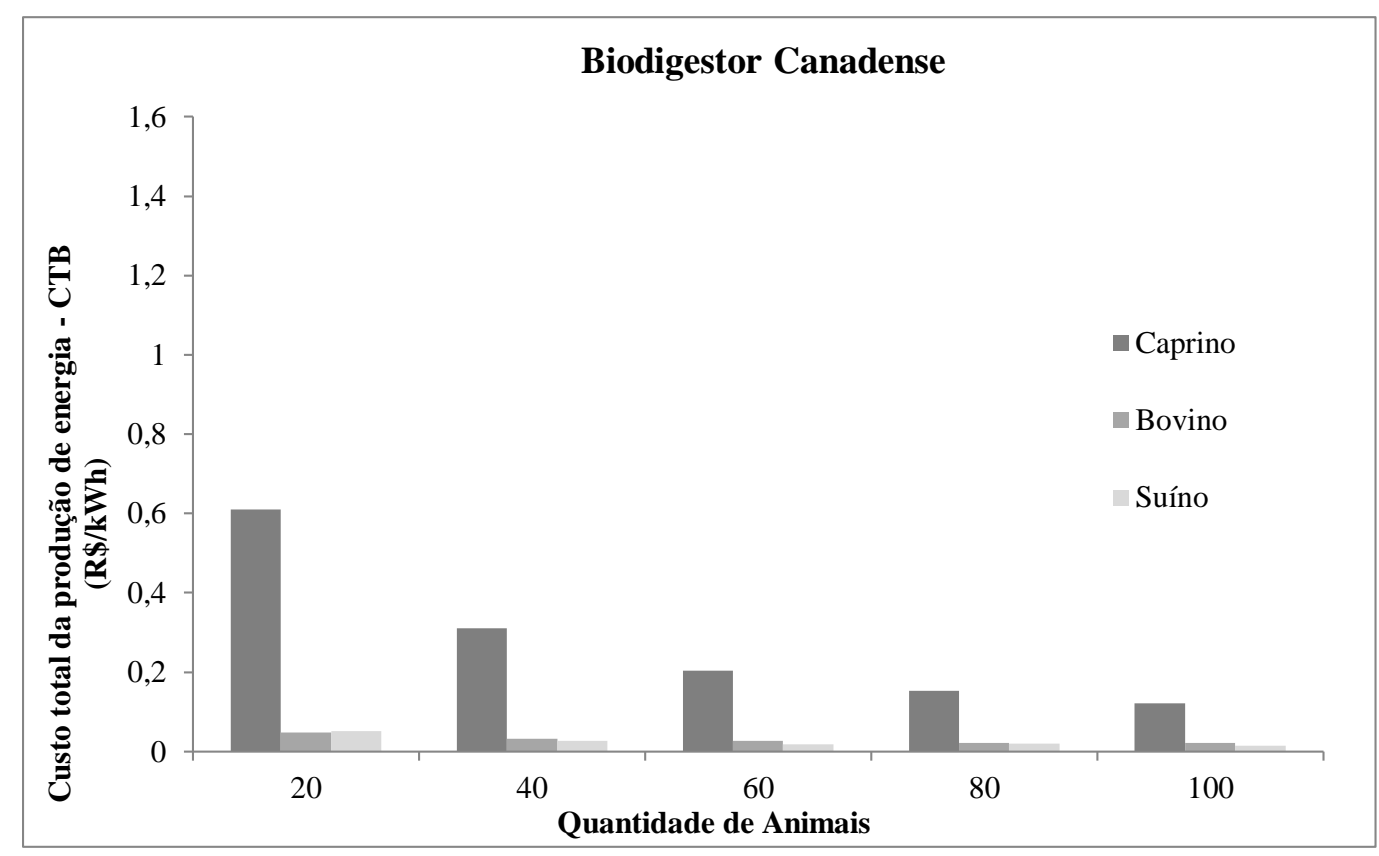

FIGURA 3. Custo total da produção de energia a partir do biogás (CTB) para biodigestor modelo Canadense. Biogas-produced energy total costs (CTB) for Canadian biodigester model.

A relação observada entre quantidade de animais e o custo total da produção de energia verifica-se para todos os modelos de biodigestor, em que, quanto maior o número de animais produzindo os dejetos para os biodigestores, menor o custo total da produção de energia a partir do biogás. 


\section{CONCLUSÕES}

Avaliaram-se os custos para a construção e implantação de biodigestores modelos chinês, indiano e canadense na cidade de Cascavel - PR, as sim como o custo total da prod ução de energia a partir do biogás em propriedades rurais com 20; 40; 60; 80 e 100 animais (caprinos, bovinos e suínos).

O biodigestor modelo canadense demonstrou ter o menor custo de construção e operação, sendo a opção mais viável para produção de energia a partir do biogás de dejetos de caprinos, bovinos e suínos, independentemente da quantidade de animais. $\mathrm{O}$ custo para este biodigestor variou de $\mathrm{R} \$ 2.104,00$ a $\mathrm{R} \$ 7.266,00$, dependendo de sua capacidade.

A geração de energia com dejetos de suínos apresentou o menor custo de produção de energia, para os três grupos de animais estudados.

A geração de energia com dejetos de caprinos apresentou o maior custo de produção de energia, para os três grupos de animais estudados.

A maior produção anual de energia, a partir do biogás, foi observada com a utilização dos dejetos bovinos, variando de $24.090 \mathrm{kWh} / \mathrm{ano}$ a $120.450 \mathrm{kWh} / \mathrm{ano}$.

\section{REFERÊNCIAS}

BALMANT, W. Concepção, construção e ope ração de um biodigestor e modelagem mate mática da biodigestão anae róbica. Dissertação (Mestrado em Processos Térmicos e Químicos) - Universidade Federal do Paraná, Curitiba, 2009.

CERVI, R. G.; ESPERANCINI, M. S. T.; BUENO, O. C. Viabilidade econômica da utilização do biogás produzido em granja suiníc ola para geração de energia elétrica. Engenharia Ag rícola, Jaboticabal, v.30, n.5, p.831-844, 2010.

DHANALAKSHMI, S. V.; RAMANUJAM, R. A. Biogas generation in a ve getable waste anaerobic digester: An analytical approach. Research Journal of Recent Sciences, v.1, n. 3, p.41-47, 2012.

FONSECA, F. S. T.; ARAÚJO, A. R. A.; HENDGES, T. L. Análise de Viabilidade Econômica de Biodigestores na Atividade Suiníc ola na Cidade de Balsas - MA: um estudo de caso. In: CONGRESSO DA SOBER, 47., Porto Ale gre, 2009. Anais...

LUCAS JÚNIOR, J.; SOUZA, C. F.; LOPES, J. D. S. Construção e operação de biodigestores, Viç osa: CPT, 176p, 2003.

NISHIMURA, R. Análise de balanço energético de sistema de produção de biogás em granja de suínos: implementação de aplicativo computacional. Dissertação (Mestrado em Engenharia Elétrica) - Universida de Fe deral de Mato Grosso do Sul, Campo Grande, 2009.

OLIVER, A. P. M.; SOUZA NETO, A. A.; QUADROS, D. G.; VALLADARES, R. E. Manual de treinamento em biodigestão. Salva dor: Instituto Winrock - Brasil, 2008.

SALOMON, K. R.; LORA. E. E. S. Estimate of the electric energy ge nerating potential for different sources of biogas in Brazil. Biomass and Bioenergy, Oxford, v.33, p.1101-1107, 2009.

SILVA, C. L.; RABELO, J. M. RAMAZZOTTE, V. C. B.; ROSSI, L. F. S.; TOMAZ, P. Curso de manejo das águas pluviais, cap.44, 5p, 2008.

SINAPI - SISTEMA NACIONAL DE PESQUISA DE CUSTOS E ÍNDICES DA CONSTRUÇÃO CIVIL. Índices da construção civil. Dis ponível em:

$<$ http://www1.caixa.gov.br/download/asp/dow nload.asp?subCategId=988\&CategId=120\&subCate glayo $\mathrm{ut}=$ Re lat $\% \mathrm{~F} 3$ rios + de + Insumos + Sete mbro\% 2F12\& Categla yout=SINAPI ++ Sist. + Nac. + Pesq. + Custos + e + Indices+Const.+Civil>. Acesso em: 21 nov. 2012.

XAVIER, C. A. N; LUCAS JÚNIOR, J. Parâmetros de dimensionamento para biodigestores batelada operados com de jetos de vacas leiteiras com e sem us o de inóculo. Engenharia Agrícola, Jaboticabal, v.30, n. 2, p.212-223, mar./abr. 2010. 